Research Article

\title{
On Some Properties of the New Generalized Fractional Derivative with Non-Singular Kernel
}

\author{
Khalid Hattaf $\mathbb{B}$ \\ Centre Régional des Métiers de L'Education et de La Formation (CRMEF), 20340 Derb Ghalef, Casablanca, Morocco \\ Correspondence should be addressed to Khalid Hattaf; k.hattaf@yahoo.fr
}

Received 6 April 2021; Accepted 19 May 2021; Published 27 May 2021

Academic Editor: Fahd Jarad

Copyright (C) 2021 Khalid Hattaf. This is an open access article distributed under the Creative Commons Attribution License, which permits unrestricted use, distribution, and reproduction in any medium, provided the original work is properly cited.

This paper presents some new formulas and properties of the generalized fractional derivative with non-singular kernel that covers various types of fractional derivatives such as the Caputo-Fabrizio fractional derivative, the Atangana-Baleanu fractional derivative, and the weighted Atangana-Baleanu fractional derivative. These new properties extend many recent results existing in the literature. Furthermore, the paper proposes some interesting inequalities that estimate the generalized fractional derivatives of some specific functions. These inequalities can be used to construct Lyapunov functions with the aim to study the global asymptotic stability of several fractional-order systems arising from diverse fields of science and engineering.

\section{Introduction}

In the literature, there exist manifold types of fractional derivatives which are used to solve several mathematical and engineering problems as well as to model the dynamics of many systems existing in various fields of science such as mechanics, chemistry, finance, ecology, biology, and control theory. For instance, Caputo and Fabrizio [1] proposed a fractional derivative with non-singular kernel to describe the material heterogeneities and the fluctuations of different scales, which cannot be well described by classical local theories or by fractional models with singular kernel. An extension of [1] was proposed by Atangana and Baleanu [2] by using Mittag-Lefler function with one parameter. Due to the importance of weighted fractional derivatives to solve several types of integral equations with elegant ways, Al-Refai [3] introduced the weighted Atangana-Baleanu fractional operators and he studied their properties. A generalized version of all previous fractional derivative operators with non-singular kernel was recently proposed in [4].

The objective of this study is to establish some properties and formulas of the new generalized fractional derivative introduced in [4] in order to extend several results presented in recent works. To achieve this goal, the remainder of this article is outlined as follows. The next section deals with preliminaries and fractional derivatives of some specific functions. The estimates of the generalized fractional derivatives of these specific functions can be applied to find Lyapunov candidate functions for demonstrating the global stability of many fractional-order systems. Section 3 is devoted to the new formulas and properties of the generalized fractional derivative with nonsingular kernel. The paper ends with a conclusion in Section 4

\section{Preliminaries and Fractional Derivatives of Some Specific Functions}

In this section, we first recall the definition and special cases of the new generalized fractional derivative with non-singular kernel. After, we present new properties for this fractional derivative that can be used to prove the global dynamics of several fractional-order systems.

Definition 1 (see [4]). Let $\alpha \in[0,1), \quad \beta, \gamma>0$, and $f \in H^{1}(a, b)$. The new generalized fractional derivative of order $\alpha$ of Caputo sense of the function $f(t)$ with respect to the weight function $w(t)$ is defined as follows: 


$$
{ }^{C} D_{a, t, w}^{\alpha, \beta, \gamma} f(t)=\frac{N(\alpha)}{1-\alpha} \frac{1}{w(t)} \int_{a}^{t} E_{\beta}\left[-\mu_{\alpha}(t-x)^{\gamma}\right] \frac{\mathrm{d}}{\mathrm{d} x}(w f)(x) \mathrm{d} x
$$

where $w \in C^{1}(a, b), w, w^{\prime}>0$ on $a, b, N(\alpha)$ is a normalization function obeying $N(0)=N(1)=1, \mu_{\alpha}=\alpha /(1-\alpha)$, and $E_{\beta}(t)=\sum_{k=0}^{+\infty}\left(t^{k} / \Gamma(\beta k+1)\right)$ is the Mittag-Leffler function of parameter $\beta$.

The importance of the fractional derivative given in the above definition is the generalization of most cases existing in the literature. These particular cases can be summarized in the following remark.

Remark 1.

(i) If $w(t)=1$ and $\beta=\gamma=1$, then (1) is reduced to the Caputo-Fabrizio fractional derivative [1] given by ${ }^{C} D_{a, t, 1}^{\alpha, 1,1} f(t)=\frac{N(\alpha)}{1-\alpha} \int_{a}^{t} \exp \left[-\mu_{\alpha}(t-x)\right] f^{\prime}(x) \mathrm{d} x$.

(ii) If $w(t)=1$ and $\beta=\gamma=\alpha$, then (1) is reduced to the Atangana-Baleanu fractional derivative [2] given by

$$
{ }^{C} D_{a, t, 1}^{\alpha, \alpha, \alpha} f(t)=\frac{N(\alpha)}{1-\alpha} \int_{a}^{t} E_{\alpha}\left[-\mu_{\alpha}(t-x)^{\alpha}\right] f^{\prime}(x) \mathrm{d} x .
$$

(iii) If $\beta=\gamma=\alpha$, then (1) is reduced to the weighted Atangana-Baleanu fractional derivative [3] given by

$$
\begin{aligned}
{ }^{C} D_{a, t, w}^{\alpha, \alpha, \alpha} f(t)= & \frac{N(\alpha)}{1-\alpha} \frac{1}{w(t)} \int_{a}^{t} E_{\alpha}\left[-\mu_{\alpha}(t-x)^{\alpha}\right] \\
& \cdot \frac{\mathrm{d}}{\mathrm{d} x}(w f)(x) \mathrm{d} x .
\end{aligned}
$$

Now, we establish new properties for the generalized fractional derivative. We begin with the following result.

Theorem 1. Let $f$ be a continuous function and $u$ be $a$ continuously differentiable function. For any constant $c$, the new generalized fractional derivative of the function $F$ defined by

$$
F(t)=\frac{1}{w(t)} \int_{c}^{w(t) u(t)} f(x) \mathrm{d} x
$$

satisfies the following property:

$$
\begin{aligned}
{ }^{C} D_{a, t, w}^{\alpha, \beta, \gamma} F(t)= & f(w(t) u(t))^{C} D_{a, t, w}^{\alpha, \beta, \gamma} u(t)-\frac{N(\alpha)}{1-\alpha} \frac{v(a)}{w(t)} E_{\beta}\left[-\mu_{\alpha}(t-a)^{\gamma}\right] \\
& -\frac{\alpha \gamma N(\alpha)}{(1-\alpha)^{2}} \frac{1}{w(t)} \int_{a}^{t}(t-\tau)^{\gamma-1} E_{\beta, \beta+1}^{2}\left[-\mu_{\alpha}(t-\tau)^{\gamma}\right] v(\tau) \mathrm{d} \tau,
\end{aligned}
$$

where

$$
\begin{aligned}
v(\tau)= & f(w(t) u(t))(w(t) u(t)-w(\tau) u(\tau)) \\
& +\int_{w(t) u(t)}^{w(\tau) u(\tau)} f(x) \mathrm{d} x .
\end{aligned}
$$

Moreover, we have the following inequalities:

(i) If $f$ is a increasing function, then

$$
{ }^{C} D_{a, t, w}^{\alpha, \beta, \gamma} F(t) \leq f(w(t) u(t)){ }^{C} D_{a, t, w}^{\alpha, \beta, \gamma} u(t) .
$$

(ii) If $f$ is a decreasing function, then

$$
{ }^{C} D_{a, t, w}^{\alpha, \beta, \gamma} F(t) \geq f(w(t) u(t))^{C} D_{a, t, w}^{\alpha, \beta, \gamma} u(t) .
$$

Proof. By using Definition 1, we have

$$
\begin{aligned}
{ }^{C} D_{a, t, w}^{\alpha, \beta, \gamma} F(t)= & \frac{N(\alpha)}{1-\alpha} \frac{1}{w(t)} \int_{a}^{t} E_{\beta} \\
& \cdot\left[-\mu_{\alpha}(t-\tau)^{\gamma}\right] f(w(\tau) u(\tau))(w u)^{\prime}(\tau) \mathrm{d} \tau .
\end{aligned}
$$

Let

$$
g(t)={ }^{C} D_{a, t, w}^{\alpha, \beta, \gamma} F(t)-f(w(t) u(t)){ }^{C} D_{a, t, w}^{\alpha, \beta, \gamma} u(t) .
$$

Then,

$$
\begin{aligned}
g(t)= & \frac{N(\alpha)}{1-\alpha} \frac{1}{w(t)} \int_{a}^{t} E_{\beta}\left[-\mu_{\alpha}(t-\tau)^{\gamma}\right](f(w(\tau) u(\tau)) \\
& -f(w(t) u(t)))(w u)^{\prime}(\tau) \mathrm{d} \tau
\end{aligned}
$$

Obviously, $\quad v^{\prime}(\tau)=(f(w(\tau) u(\tau))-f(w(t) u(t)))$ $(w u)^{\prime}(\tau)$ and $v(t)=0$. Integrating by parts the last integral, we find

$$
\begin{aligned}
g(t)= & \left.\frac{N(\alpha)}{1-\alpha} \frac{1}{w(t)} E_{\beta}\left[-\mu_{\alpha}(t-\tau)^{\gamma}\right] v(\tau)\right|_{\tau=a} ^{\tau=t} \\
= & \frac{\alpha \gamma N(\alpha)}{(1-\alpha)^{2}} \frac{1}{w(t)} \int_{a}^{t}(t-\tau)^{\gamma-1} E_{\beta, \beta+1}^{2} \\
& \cdot\left[-\mu_{\alpha}(t-\tau)^{\gamma}\right] v(\tau) \mathrm{d} \tau .
\end{aligned}
$$




$$
\begin{aligned}
g(t)= & -\frac{N(\alpha)}{1-\alpha} \frac{v(a)}{w(t)} E_{\beta}\left[-\mu_{\alpha}(t-a)^{\gamma}\right] \\
& -\frac{\alpha \gamma N(\alpha)}{(1-\alpha)^{2}} \frac{1}{w(t)} \int_{a}^{t}(t-\tau)^{\gamma-1} E_{\beta, \beta+1}^{2} \\
& \cdot\left[-\mu_{\alpha}(t-\tau)^{\gamma}\right] v(\tau) \mathrm{d} \tau .
\end{aligned}
$$

Now, we consider the following function:

$$
\psi_{c, f}(\tau)=f(c)(c-\tau)+\int_{c}^{\tau} f(x) \mathrm{d} x .
$$

It is clear that $\psi_{c, f}^{\prime}(\tau)=f(\tau)-f(c)$. If $f$ is a increasing function, then the function $\psi_{c, f}(\tau)$ is decreasing on the interval $(-\infty, c]$ and increasing on $[c,+\infty)$ with $\psi_{c, f}(c)=0$. Hence, $\psi_{c, f}(\tau)$ has the global minimum at $\tau=c$. So,

$$
\psi_{c, f}(\tau) \geq 0, \quad \text { for all }(c, \tau) \in \mathrm{IR}^{2} .
$$

Since $v(\tau)=\psi_{w(t) u(t), f}(w(\tau) u(\tau))$, we have $v(\tau) \geq 0$ for all $\tau \in$ IR. This proves (i). In the same way, we can easily prove (ii).

Corollary 1. Let $u(t) \in I R$ be a continuously differentiable function. Then, for any time $t \geq t_{0}$, we have

$$
{ }^{C} D_{t_{0}, t, 1}^{\alpha, \beta, \gamma} u^{2}(t) \leq 2 u(t){ }^{C} D_{t_{0}, t, 1}^{\alpha, \beta, \gamma} u(t)
$$

Proof. Applying (i) of Theorem 1 to the function $f(x)=2 x$, we get

$$
\begin{aligned}
{ }^{C} D_{t_{0}, t, 1}^{\alpha, \beta, \gamma} u^{2}(t) & ={ }^{C} D_{t_{0}, t, 1}^{\alpha, \beta, \gamma} \int_{c}^{u(t)} 2 x \mathrm{~d} x \\
& \leq 2 u(t)^{C} D_{t_{0}, t, 1}^{\alpha, \beta, \gamma} u(t) .
\end{aligned}
$$

This completes the proof.

Remark 2. Corollary 1 extends the result presented in Lemma 3.1 of [5] for the new generalized fractional derivative. In fact, it suffices to take $\gamma=\beta=\alpha$ in inequality (17).

Now, we extend the recent result presented in Lemma 3.2 of [5] by proposing the following corollary.

Corollary 2. Let $u(t) \in I R^{+}$be a continuously differentiable function and $u^{*}>0$. Then, for any time $t \geq t_{0}$, we have

$$
{ }^{C} D_{t_{0}, t, 1}^{\alpha, \beta, \gamma}\left[u(t)-u^{*}-u^{*} \ln \frac{u(t)}{u^{*}}\right] \leq\left(1-\frac{u^{*}}{u(t)}\right){ }^{C} D_{t_{0}, t, 1}^{\alpha, \beta, \gamma} u(t) .
$$

Proof. The application of Theorem 1 (ii) to the function $f(x)=1 / x$ leads to the following:

$$
\begin{gathered}
{ }^{C} D_{t_{0}, t, 1}^{\alpha, \beta, \gamma}\left[u(t)-u^{*}-u^{*} \ln \frac{u(t)}{u^{*}}\right] \\
={ }^{C} D_{t_{0}, t, 1}^{\alpha, \beta, \gamma} u(t)-u^{* C} D_{t_{0}, t, 1}^{\alpha, \beta, \gamma} \int_{u^{*}}^{u(t)} \frac{1}{x} \mathrm{~d} x \\
\leq\left(1-\frac{u^{*}}{u(t)}\right){ }^{C} D_{t_{0}, t, 1}^{\alpha, \beta, \gamma} u(t) .
\end{gathered}
$$

The proof of Corollary 2 is completed.

\section{New Formulas and Properties}

For simplicity, we denote ${ }^{C} D_{a, t, 1}^{\alpha, \beta, \beta}$ by $\mathscr{D}_{a, w}^{\alpha, \beta}$. According to [4], we have the following definition.

Definition 2 (see [4]). The generalized fractional integral operator corresponding to $\mathscr{D}_{a, w}^{\alpha, \beta}$ is defined by

$$
\mathscr{I}_{a, w}^{\alpha, \beta} f(t)=\frac{1-\alpha}{N(\alpha)} f(t)+\frac{\alpha}{N(\alpha)} R L \mathscr{F}_{a, w}^{\beta} f(t),
$$

where ${ }^{R L} \mathscr{I}_{a, w}^{\beta}$ is the standard weighted Riemann-Liouville fractional integral of order $\beta$ defined by

$$
{ }^{R L} \mathscr{J}_{a, w}^{\beta} f(t)=\frac{1}{\Gamma(\beta)} \frac{1}{w(t)} \int_{a}^{t}(t-x)^{\beta-1} w(x) f(x) \mathrm{d} x .
$$

Remark 3. The Atangana-Baleanu fractional integral operator is a special case of (21), and it suffices to take $w(t)=1$ and $\beta=\alpha$.

Lemma 1. The fractional derivative $\mathscr{D}_{a, w}^{\alpha, \beta}$ can be expressed as follows:

$$
\mathscr{D}_{a, w}^{\alpha, \beta} f(t)=\frac{N(\alpha)}{1-\alpha} \sum_{k=0}^{+\infty}\left(-\mu_{\alpha}\right)^{k R L} \mathscr{G}_{a, w}^{\beta k+1}\left(\frac{(w f)^{\prime}}{w}\right)(t) .
$$

This series converges locally and uniformly in $t$ for any a, $\alpha, \beta, w$, and $f$ satisfying the conditions from Definition 1 .

Proof. The Mittag-Leffler function $E_{\alpha}(t)$ is a sum of an entire series. This series converges locally and uniformly in the whole complex plane. Hence, we can rewrite the fractional derivative $\mathscr{D}_{a, w}^{\alpha, \beta}$ as follows:

$$
\begin{aligned}
\mathscr{D}_{a, w}^{\alpha, \beta} f(t)= & \frac{N(\alpha)}{1-\alpha} \frac{1}{w(t)} \sum_{k=0}^{+\infty} \frac{\left(-\mu_{\alpha}\right)^{k}}{\Gamma(\beta k+1)} \\
& \cdot \int_{a}^{t}(t-x)^{\beta k}(w f)^{\prime}(x) \mathrm{d} x \\
= & \frac{N(\alpha)}{1-\alpha} \sum_{k=0}^{+\infty}\left(-\mu_{\alpha}\right)^{k} \frac{1}{\Gamma(\beta k+1)} \frac{1}{w(t)} \\
& \cdot \int_{a}^{t}(t-x)^{\beta k}(w f)^{\prime}(x) \mathrm{d} x \\
= & \frac{N(\alpha)}{1-\alpha} \sum_{k=0}^{+\infty}\left(-\mu_{\alpha}\right)^{k R L} \mathscr{g}_{a, w}^{\beta k+1}\left(\frac{(w f)^{\prime}}{w}\right)(t) .
\end{aligned}
$$


This completes the proof.

(ii) $\mathscr{I}_{a, w}^{\alpha, \beta}\left(\mathscr{I}_{a, w}^{\gamma, \rho} f\right)(t)=\mathscr{I}_{a, w}^{\gamma, \rho}\left(\mathscr{I}_{a, w}^{\alpha, \beta} f\right)(t)$.

Theorem 2. Let $\alpha, \gamma \in[0,1), \beta, \rho>0$, and $f \in H^{1}(a, b)$. Then,

(i) $\mathscr{D}_{a, w}^{\alpha, \beta}\left(\mathscr{D}_{a, w}^{\gamma, \rho} f\right)(t)=\mathscr{D}_{a, w}^{\gamma, \rho}\left(\mathscr{D}_{a, w}^{\alpha, \beta} f\right)(t)$.
This means that the generalized fractional integrals and derivatives are commutative operators.

Proof. First, we prove (i). By applying Lemma 1, we get

$$
\begin{aligned}
\mathscr{D}_{a, w}^{\alpha, \beta}\left(\mathscr{D}_{a, w}^{\gamma, \rho} f\right)(t) & =\frac{N(\alpha)}{1-\alpha} \sum_{k=0}^{+\infty}\left(-\mu_{\alpha}\right)^{k R L} \mathscr{J}_{a, w}^{\beta k+1}\left(\frac{\left(w \mathscr{D}_{a, w}^{\gamma, \rho} f\right)^{\prime}}{w}\right)(t) \\
& =\frac{N(\alpha)}{1-\alpha} \sum_{k=0}^{+\infty}\left(-\mu_{\alpha}\right)^{k R L} \mathscr{J}_{a, w}^{\beta k+1}\left[\frac{N(\gamma)}{1-\gamma} \sum_{n=0}^{+\infty}\left(-\mu_{\gamma}\right)^{n R L} \mathscr{F}_{a, w}^{\rho n}\left(\frac{(w f)^{\prime}}{w}\right)\right](t) \\
& =\frac{N(\alpha) N(\gamma)}{(1-\alpha)(1-\gamma)} \sum_{k=0}^{+\infty} \sum_{n=0}^{+\infty}\left(-\mu_{\alpha}\right)^{k}\left(-\mu_{\gamma}\right)^{n_{R L}} \mathscr{J}_{a, w}^{\beta k+\rho n+1}\left(\frac{(w f)^{\prime}}{w}\right)(t) .
\end{aligned}
$$

Since the last expression is symmetric in $\alpha$ and $\gamma$ and also in $\beta$ and $\rho$, we easily deduce (i).
For (ii), we have

$$
\begin{aligned}
& \mathscr{I}_{a, w}^{\alpha, \beta}\left(\mathscr{J}_{a, w}^{\gamma, \rho} f\right)(t)=\frac{1-\alpha}{N(\alpha)}\left(\mathscr{I}_{a, w}^{\gamma, \rho} f\right)(t)+\frac{\alpha}{N(\alpha)}{ }^{R L} \mathscr{I}_{a, w}^{\beta}\left(\mathscr{I}_{a, w}^{\gamma, \rho} f\right)(t) \\
& =\frac{1-\alpha}{N(\alpha)}\left(\frac{1-\gamma}{N(\gamma)} f(t)+\frac{\gamma}{N(\gamma)} R L \mathscr{F}_{a, w}^{\rho} f(t)\right) \\
& +\frac{\alpha}{N(\alpha)} R L \mathscr{J}_{a, w}^{\beta}\left(\frac{1-\gamma}{N(\gamma)} f(t)+\frac{\gamma}{N(\gamma)}{ }^{R L} \mathscr{I}_{a, w}^{\rho} f(t)\right) \\
& =\frac{(1-\alpha)(1-\gamma)}{N(\alpha) N(\gamma)} f(t)+\frac{\gamma(1-\alpha)}{N(\alpha) N(\gamma)} R L \mathscr{F}_{a, w}^{\rho} f(t) \\
& +\frac{\alpha(1-\gamma)}{N(\alpha) N(\gamma)} R L \mathscr{J}_{a, w}^{\beta} f(t)+\frac{\alpha \gamma}{N(\alpha) N(\gamma)}{ }^{R L} \mathscr{J}_{a, w}^{\beta+\rho} f(t) \\
& =\mathscr{I}_{a, w}^{\gamma, \rho}\left(\mathscr{J}_{a, w}^{\alpha, \beta} f\right)(t)
\end{aligned}
$$

This shows (ii).

(ii) $\mathscr{D}_{a, w}^{\alpha, \beta}\left(\mathscr{I}_{a, w}^{\alpha, \beta} f\right)(t)=f(t)-((w(a) f(a)) / w(t))$.

Theorem 3. Let $\alpha \in[0,1), \beta>0$, and $f \in H^{1}(a, b)$. Then,

(i) $\mathscr{I}_{a, w}^{\alpha, \beta}\left(\mathscr{D}_{a, w}^{\alpha, \beta} f\right)(t)=f(t)-((w(a) f(a)) / w(t))$.

Proof. According Definition 2 and Lemma 1, we have

$$
\begin{aligned}
\mathscr{I}_{a, w}^{\alpha, \beta}\left(\mathscr{D}_{a, w}^{\alpha, \beta} f\right)(t) & =\frac{1-\alpha}{N(\alpha)}\left(\mathscr{D}_{a, w}^{\alpha, \beta} f\right)(t)+\frac{\alpha}{N(\alpha)}{ }^{R L} \mathscr{J}_{a, w}^{\beta}\left(\mathscr{D}_{a, w}^{\alpha, \beta} f\right)(t) \\
& =\frac{1-\alpha}{N(\alpha)}\left[\frac{N(\alpha)}{1-\alpha} \sum_{k=0}^{+\infty}\left(-\mu_{\alpha}\right)^{k R L} \mathscr{I}_{a, w}^{\beta k+1}\left(\frac{(w f)^{\prime}}{w}\right)(t)\right]
\end{aligned}
$$




$$
\begin{aligned}
& +\frac{\alpha}{N(\alpha)}{ }^{R L} \mathscr{I}_{a, w}^{\beta}\left[\frac{N(\alpha)}{1-\alpha} \sum_{k=0}^{+\infty}\left(-\mu_{\alpha}\right)^{k R L} \mathscr{J}_{a, w}^{\beta k+1}\left(\frac{(w f)^{\prime}}{w}\right)\right](t) \\
= & \sum_{k=0}^{+\infty}\left(-\mu_{\alpha}\right)^{k R L} \mathscr{J}_{a, w}^{\beta k+1}\left(\frac{(w f)^{\prime}}{w}\right)(t)-\sum_{k=0}^{+\infty}\left(-\mu_{\alpha}\right)^{k+1 R L} \mathscr{J}_{a, w}^{\beta(k+1)+1}\left(\frac{(w f)^{\prime}}{w}\right)(t) .
\end{aligned}
$$

Hence,

$$
\begin{aligned}
\mathscr{F}_{a, w}^{\alpha, \beta}\left(\mathscr{D}_{a, w}^{\alpha, \beta} f\right)(t) & =\sum_{k=0}^{+\infty}\left(-\mu_{\alpha}\right)^{k R L} \mathscr{F}_{a, w}^{\beta k+1}\left(\frac{(w f)^{\prime}}{w}\right)(t)-\sum_{k=1}^{+\infty}\left(-\mu_{\alpha}\right)^{k R L} \mathscr{I}_{a, w}^{\beta k+1}\left(\frac{(w f)^{\prime}}{w}\right)(t) \\
& ={ }^{R L} \mathscr{I}_{a, w}^{1}\left(\frac{(w f)^{\prime}}{w}\right)(t) \\
& =\frac{1}{w(t)} \int_{a}^{t}(w f)^{\prime}(x) \mathrm{d} x=\frac{1}{w(t)}(w(t) f(t)-w(a) f(a)) .
\end{aligned}
$$

Now, we demonstrate (ii). From Lemma 1, we obtain

$$
\begin{aligned}
& \mathscr{D}_{a, w}^{\alpha, \beta}\left(\mathscr{I}_{a, w}^{\alpha, \beta} f\right)(t)=\frac{N(\alpha)}{1-\alpha} \sum_{k=0}^{+\infty}\left(-\mu_{\alpha}\right)^{k R L} \mathscr{F}_{a, w}^{\beta k+1}\left(\frac{\left(w \mathscr{F}_{a, w}^{\alpha, \beta} f\right)^{\prime}}{w}\right)(t) \\
& =\frac{N(\alpha)}{1-\alpha} \sum_{k=0}^{+\infty}\left(-\mu_{\alpha}\right)^{k R L} \mathscr{I}_{a, w}^{\beta k+1}\left[\frac{1-\alpha}{N(\alpha)} \frac{(w f)^{\prime}}{w}+\frac{\alpha}{N(\alpha)} \frac{\left(w^{R L} \mathscr{I}_{a, w}^{\beta} f\right)^{\prime}}{w}\right](t) \\
& =\sum_{k=0}^{+\infty}\left(-\mu_{\alpha}\right)^{k R L} \mathscr{J}_{a, w}^{\beta k+1}\left(\frac{(w f)^{\prime}}{w}\right)(t) \\
& +\mu_{\alpha} \sum_{k=0}^{+\infty}\left(-\mu_{\alpha}\right)^{k R L} \mathscr{I}_{a, w}^{\beta k+1}\left(\frac{\left(w^{R L} \mathscr{J}_{a, w}^{\beta} f\right)^{\prime}}{w}\right)(t) \\
& =\sum_{k=0}^{+\infty}\left(-\mu_{\alpha}\right)^{k}\left[R L \mathscr{F}_{a, w}^{\beta k} f(t)-(w f)(a)^{R L} \mathscr{J}_{a, w}^{\beta k}\left(\frac{1}{w}\right)(t)\right]
\end{aligned}
$$

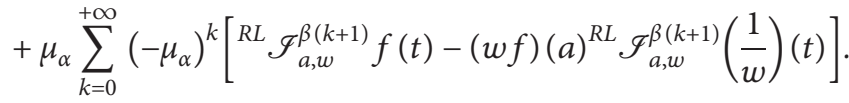

Thus,

$$
\begin{aligned}
\mathscr{D}_{a, w}^{\alpha, \beta}\left(\mathscr{I}_{a, w}^{\alpha, \beta} f\right)(t)= & \sum_{k=0}^{+\infty}\left(-\mu_{\alpha}\right)^{k}\left[R L \mathscr{J}_{a, w}^{\beta k} f(t)-(w f)(a)^{R L} \mathscr{G}_{a, w}^{\beta k}\left(\frac{1}{w}\right)(t)\right] \\
& -\sum_{k=1}^{+\infty}\left(-\mu_{\alpha}\right)^{k}\left[{ }^{R L} \mathscr{J}_{a, w}^{\beta k} f(t)-(w f)(a)^{R L} \mathscr{J}_{a, w}^{\beta k}\left(\frac{1}{w}\right)(t)\right] \\
= & { }^{R L} \mathscr{I}_{a, w}^{0} f(t)-(w f)(a)^{R L} \mathscr{J}_{a, w}^{0}\left(\frac{1}{w}\right)(t) \\
= & f(t)-\frac{(w f)(a)}{w(t)} .
\end{aligned}
$$


This completes the proof.

By applying Theorem 3 for $w(t)=1$, we get the following important result that extends the Newton-Leibniz formula presented in $[6,7]$.

Corollary 3. The generalized fractional integral and the generalized fractional derivative satisfy the following Newton-Leibniz formula:

$$
\mathscr{F}_{a, 1}^{\alpha, \beta}\left(\mathscr{D}_{a, 1}^{\alpha, \beta} f\right)(t)=\mathscr{D}_{a, 1}^{\alpha, \beta}\left(\mathscr{I}_{a, 1}^{\alpha, \beta} f\right)(t)=f(t)-f(a) .
$$

\section{Conclusion}

In many fields of science like mathematical biology, the method of constructing Lyapunov functions is often based on quadratic and Volterra-type functions. So, the first aim of this work was to estimate the generalized fractional derivatives of these specific functions. The obtained results extend many elementary lemmas existing in the recent literature for other types of fractional derivatives. Furthermore, the second purpose of this work was to establish a new formula for the generalized fractional derivative in the form of a series of weighted Riemann-Liouville fractional integrals. By means of this formula, various properties of the generalized fractional derivatives and integrals operators have been carefully established. Moreover, the Newton-Leibniz formula has been rigorously extended.

The results presented in this study form a fundamental basis for extending the classical Lyapunov theorems [8-10] and LaSalle's invariance principle [11] to the case of the new generalized fractional derivative. This issue will be the first direction of our future works.

\section{Data Availability}

The data used to support the findings of this study are available from the corresponding author upon request.

\section{Conflicts of Interest}

The author declares that he has no conflicts of interest.

\section{References}

[1] A. Caputo and M. Fabrizio, "A new definition of fractional derivative without singular kernel," Progress in Fractional Differentiation and Applications, vol. 1, pp. 73-85, 2015.

[2] A. Atangana and D. Baleanu, "New fractional derivatives with nonlocal and non-singular kernel: theory and application to heat transfer model," Thermal Science, vol. 20, no. 2, pp. 763-769, 2016.

[3] M. Al-Refai, "On weighted Atangana-Baleanu fractional operators," Advances in Difference Equations, vol. 2020, pp. 1-11, 2020.

[4] K. Hattaf, "A new generalized definition of fractional derivative with non-singular kernel," Computation, vol. 8, pp. 1-9, 2020.

[5] M. A. Taneco-Hernández and C. Vargas-De-León, "Stability and Lyapunov functions for systems with Atangana-Baleanu Caputo derivative: an HIV/AIDS epidemic model," Chaos, Solitons and Fractals, vol. 132, Article ID 109586, 2020.
[6] J. D. Diida, A. Atangana, and I. Area, "Numerical computation of a fractional derivative with non-local and non-singular kernel," Mathematical Modelling of Natural Phenomena, vol. 12, no. 3, pp. 4-13, 2017.

[7] D. Baleanu and A. Fernandez, "On some new properties of fractional derivatives with Mittag-Leffler kernel," Communications in Nonlinear Science and Numerical Simulation, vol. 59, pp. 444-462, 2018.

[8] A. M. Lyapunov, "The general problem of the stability of motion," International Journal of Control, vol. 55, no. 3, pp. 531-534, 1992.

[9] H. K. Khalil, Nonlinear Systems, Prentice-Hall, Englewood, NY, USA, Third edition, 2002.

[10] K. Hattaf and N. Yousfi, "Global stability for reaction-diffusion equations in biology," Computers \& Mathematics with Applications, vol. 66, no. 8, pp. 1488-1497, 2013.

[11] J. P. LaSalle, The Stability of Dynamical Systems, Regional Conference Series in Applied Mathematics, SIAM Philadelphia, Philadelphia, PA, USA, 1976. 